\title{
Perspective Piece \\ Effect of SARS-CoV-2 Infection in Pregnancy on Maternal and Neonatal Outcomes in Africa: An AFREhealth Call for Evidence through Multicountry Research Collaboration
}

Jean B. Nachega, ${ }^{1,2,3,4 *}$ † Nadia A. Sam-Agudu, ${ }^{5,6,7}$ † Samantha Budhram, ${ }^{8}$ Taha E. Taha, ${ }^{3}$ Valerie Vannevel, ${ }^{9}$ Priya Somapillay, ${ }^{10}$ Daniel Katuashi Ishoso, ${ }^{11}$ Michel Tshiasuma Pipo, ${ }^{12}$ Christian Bongo-Pasi Nswe, ${ }^{12,13}$ John Ditekemena, ${ }^{11}$ Birhanu T. Ayele, ${ }^{14}$ Rhoderick N. Machekano, ${ }^{14}$ Onesmus W. Gachuno, ${ }^{15}$ John Kinuthia, ${ }^{15}, 16$ Nancy Mwongeli, ${ }^{16}$ Musa Sekikubo, ${ }^{17}$ Philippa Musoke, ${ }^{18}$ Evans Kofi Agbeno, ${ }^{19}$ Lawal W. Umar, ${ }^{20}$ Mukanire Ntakwinja, ${ }^{21}$ Denis M. Mukwege, ${ }^{21}$ Emily R. Smith, ${ }^{22}$ Eduard J. Mills, ${ }^{23}$ John Otokoye Otshudiema, ${ }^{24}$ Placide Mbala-Kingebeni, ${ }^{14}$ Jean-Marie N. Kayembe, ${ }^{25}$ Don Jethro Mavungu Landu, ${ }^{12,13}$ Jean-Jacques Muyembe Tamfum, ${ }^{26}$ Alimuddin Zumla, ${ }^{27,28}$ Eduard J. Langenegger, ${ }^{29}$ and Lynne M. Mofenson ${ }^{30}$ for the AFREhealth COVID-19 Research Collaboration Working Group

${ }^{1}$ Department of Medicine, Stellenbosch University, Cape Town, South Africa; ${ }^{2}$ Department of Epidemiology, Infectious Diseases and Microbiology, Center for Global Health, University of Pittsburgh, Pittsburgh, Pennsylvania; ${ }^{3}$ Department of Epidemiology, Johns Hopkins Bloomberg School of Public Health, Baltimore, Maryland; ${ }^{4}$ Department of International Health, Bloomberg School of Public Health, Johns Hopkins University, Baltimore, Maryland; ${ }^{5}$ International Research Center of Excellence, Department of Pediatrics and Institute of Human Virology Nigeria, Abuja, Nigeria; ${ }^{6}$ Department of Pediatrics, Institute of Human Virology, University of Maryland School of Medicine, Baltimore, Maryland; ${ }^{7}$ Department of Paediatrics and Child Health, University of Cape Coast School of Medical Sciences, Cape Coast, Ghana; ${ }^{8}$ Department of Obstetrics and Gynecology, University of KwaZulu Natal, Durban, South Africa; ${ }^{9}$ UP/SAMRC Maternal and Infant Health Care Strategies Unit, Department of Obstetrics and Gynecology, Kalafong Hospital, University of Pretoria, Pretoria, South Africa; ${ }^{10}$ Maternal Foetal Medicine, Steve Biko Hospital, University of Pretoria, Pretoria, South Africa; ${ }^{11}$ Department of Community Health, School of Public Health, University of Kinshasa, Kinshasa, Democratic Republic of the Congo; ${ }^{12}$ Faculty of Public Health, Université Moderne de Kinkole, Kinshasa, Democratic Republic of Congo; ${ }^{13}$ Department of Public Health, Faculty of Medicine, Centre Interdisciplinaire de Recherche en Ethnopharmacologie, Université Notre-Dame du Kasayi, Kananga, Democratic Republic of Congo; ${ }^{14}$ Division of Epidemiology and Biostatistics, Department of Global Health, Faculty of Medicine and Health Sciences, Stellenbosch University, Cape Town, South Africa; ${ }^{15}$ Department of Obstetrics and Gynaecology, University of Nairobi, Nairobi, Kenya; ${ }^{16}$ Department of Research, Department of Reproductive Health, Kenyatta National Hospital, Nairobi, Kenya; ${ }^{17}$ Department of Obstetrics and Gynaecology, School of Medicine, College of Health Sciences, Makerere University, Kampala, Uganda; ${ }^{18}$ Department of Paediatrics and Child Health, School of Medicine, College of Health Sciences, Makerere University, Kampala, Uganda; ${ }^{19}$ Department of Obstetrics and Gynecology, Cape Coast Teaching Hospital, University of Cape Coast, Cape Coast, Ghana; ${ }^{20}$ Department of Pediatrics, College of Health Sciences, Ahmadu Bello Teaching Hospital, Ahmadu Bello University, Zaria, Nigeria; ${ }^{21}$ Gynaecology and General Surgery, Panzi General Referral Hospital, Bukavu, Democratic Republic of the Congo; ${ }^{22}$ Department of Global Health, Milken Institute School of Public Health, George Washington University, Washington, District of Columbia; ${ }^{23}$ Department of Health Research Evidence and Impact, Faculty of Health Sciences, McMaster University, Hamilton, Canada; ${ }^{24}$ Epidemiological Surveillance Team, COVID-19 Response, Health Emergencies Program, World Health Organization, Kinshasa, Democratic Republic of the Congo; ${ }^{25}$ Department of Medicine, Faculty of Medicine, University of Kinshasa, Kinshasa, Democratic Republic of the Congo; ${ }^{26}$ Department of Medical Microbiology and Virology, Faculty of Medicine, National Institute of Biomedical Research (INRB), University of Kinshasa, Kinshasa, Democratic Republic of the Congo; ${ }^{27}$ Division of Infection and Immunity, Department of Infection, Centre for Clinical Microbiology, University College London, London, United Kingdom; ${ }^{28}$ National Institute for Health Research Biomedical Research Centre, University College London Hospitals, London, United Kingdom; ${ }^{29}$ Department of Obstetrics and Gynecology, Tyberberg Teaching Hospital, Stellenbosch University Faculty of Medicine and Health Sciences, Cape Town, South Africa; ${ }^{30}$ Elizabeth Glaser Pediatric AIDS Foundation, Washington, District of Columbia

\begin{abstract}
In the African context, there is a paucity of data on SARS-CoV-2 infection and associated COVID-19 in pregnancy. Given the endemicity of infections such as malaria, HIV, and tuberculosis (TB) in sub-Saharan Africa (SSA), it is important to evaluate coinfections with SARS-CoV-2 and their impact on maternal/infant outcomes. Robust research is critically needed to evaluate the effects of the added burden of COVID-19 in pregnancy, to help develop evidence-based policies toward improving maternal and infant outcomes. In this perspective, we briefly review current knowledge on the clinical features of COVID-19 in pregnancy; the risks of preterm birth and cesarean delivery secondary to comorbid severity; the effects of maternal SARS-CoV-2 infection on the fetus/neonate; and in utero mother-to-child SARS-CoV-2 transmission. We further highlight the need to conduct multicountry surveillance as well as retrospective and prospective cohort studies across SSA. This will enable assessments of SARS-CoV-2 burden among pregnant African women and improve the understanding of the spectrum of COVID-19 manifestations in this population, which may be living with or without HIV, TB, and/or other coinfections/comorbidities. In addition, multicountry studies will allow a better understanding of risk factors and outcomes to be compared across countries and subregions. Such an approach will encourage and strengthen much-needed intra-African, south-to-south multidisciplinary and interprofessional research collaborations. The African Forum for Research and Education in Health's COVID-19 Research Working Group has embarked upon such a collaboration across Western, Central, Eastern and Southern Africa.
\end{abstract}

Data on SARS-CoV-2 and associated COVID-19 in pregnancy and their effects on maternal and fetal/infant health are limited. This is especially true for sub-Saharan Africa (SSA),

* Address correspondence to Jean B. Nachega, Department of Medicine and Centre for Infectious Diseases, Stellenbosch University Faculty of Medicine and Health Sciences, Francie Van Zijl Drive, Cape Town 7505, South Africa. E-mail: jnachega@sun.ac.za

†These authors contributed equally to this work. where there is coexisting and highly prevalent HIV, tuberculosis (TB), malaria, and malnutrition, and some of the poorest maternal and child health outcomes globally., ${ }^{1,2}$ As of December 5, 2020, the WHO African region had recorded 1,529,436 COVID-19 cases and 34,125 deaths (case fatality rate: $2.2 \%) .{ }^{3}$ To the best of our knowledge, there have been no published prospective or large-scale studies evaluating the impact of SARS-CoV-2 infection on pregnancy in SSA. ${ }^{4}$ 
Comorbidities, coinfections, and socioeconomic and health system inequalities in SSA may interact with and worsen COVID-19 among pregnant African women and contribute to already poor health outcomes among these women and neonates (Figure 1). There is an urgent need to conduct epidemiological surveillance and cohort studies that evaluate the burden and effects of COVID-19 in the region, particularly among pregnant women.

Other respiratory viral illnesses in pregnancy have been associated with high rates of adverse maternal and perinatal outcomes. For example, in the United States, 23\% of pregnant women infected with influenza $\mathrm{H} 1 \mathrm{~N} 1$ required intensive care unit (ICU) admission, and $5 \%$ of all deaths due to H1N1 occurred among pregnant patients. ${ }^{5,6}$ Initial data on COVID-19 in pregnancy were derived from small case reports or case studies, first from China and then Italy and the United States, with most reporting on women infected during the third trimester; these initial reports suggested that pregnant women were not at higher risk for SARS-CoV-2 infection than nonpregnant women of similar age. ${ }^{7-10}$ Universal SARS-CoV-2 screening of women in labor in the United States and United Kingdom (UK) has shown that, as in the general population, a large proportion of SARS-CoV-2-infected pregnant women are asymptomatic..$^{9,11,12}$ Among COVID-19 symptomatic women, fever and cough are the most observed symptoms in pregnancy. ${ }^{10,13,14}$ However, pregnant women who are asymptomatic at the time of delivery may be at risk for clinical progression in the postpartum period, developing fever and respiratory symptoms. ${ }^{15,16}$

Initial data discussed previously suggested that most pregnant women with COVID-19 had asymptomatic or mild disease. However, a recent evaluation of women of reproductive age with confirmed COVID-19 in the United States revealed that pregnant women were significantly more likely to be hospitalized and require ICU admission, mechanical ventilation, and extracorporeal membrane oxygenation (ECMO) than nonpregnant women with COVID-19, after adjusting for age, underlying conditions, and race/ethnicity. ${ }^{7,14}$ Nevertheless, the risk of mortality appears low and similar between pregnant and nonpregnant women of similar age $(0.1-0.2 \%) .{ }^{7,13,14}$ As in the nonpregnant population, preexisting comorbidities, higher maternal age, and higher body mass index are risk factors for severe disease. ${ }^{7,10,13,14}$ There have been reports of severe complications of COVID-19 among pregnant women, including cardiomyopathy, coagulopathy, need for ECMO, and death. 9,17

To date, there have been few maternal/infant COVID-19 studies from SSA. In a small observational cohort of pregnant women admitted with COVID-19 in Cameroon, common presenting symptoms were fever, cough, and dyspnea; ultimately four of the 18 women died in hospital (case fatality rate: $22 \%) .{ }^{18}$ Among the 13 women who delivered during the study, eight underwent cesarean section mostly because of maternal distress, and four newborns of the 13 deliveries died in hospital; however, neonatal outcomes could not be irrefutably attributed to COVID-19. ${ }^{18}$ A second small-scale study from Senegal reported on nine pregnant women presenting largely with cough and rhinorrhea who were admitted; median time to recovery was $\sim 14$ days, and none of them died. ${ }^{19}$ Last, Nachega et al. ${ }^{20}$ described nearly 800 COVID-19 hospital admission cases from the Democratic Republic of the Congo, which included 12 pregnant women of a total of 262 women in the analysis. Three (25\%) of the pregnant women had severe disease; however, none of the 12 pregnant women died, and there were also no significant differences in severity of disease between pregnant and nonpregnant women in this COVID-19 cohort. Of note, the last two studies did not report data on postpartum maternal or neonatal outcomes.

The risk of preterm birth and cesarean delivery secondary to maternal disease severity appears increased in pregnant women with COVID-19, compared with those without COVID19 , and neonates appear more likely to be admitted to the neonatal ICU (although in some cases solely for observation of SARS-CoV-2 exposure or secondary to prematurity). ${ }^{10,13,21,22}$ In general, beyond complications of prematurity, neonatal

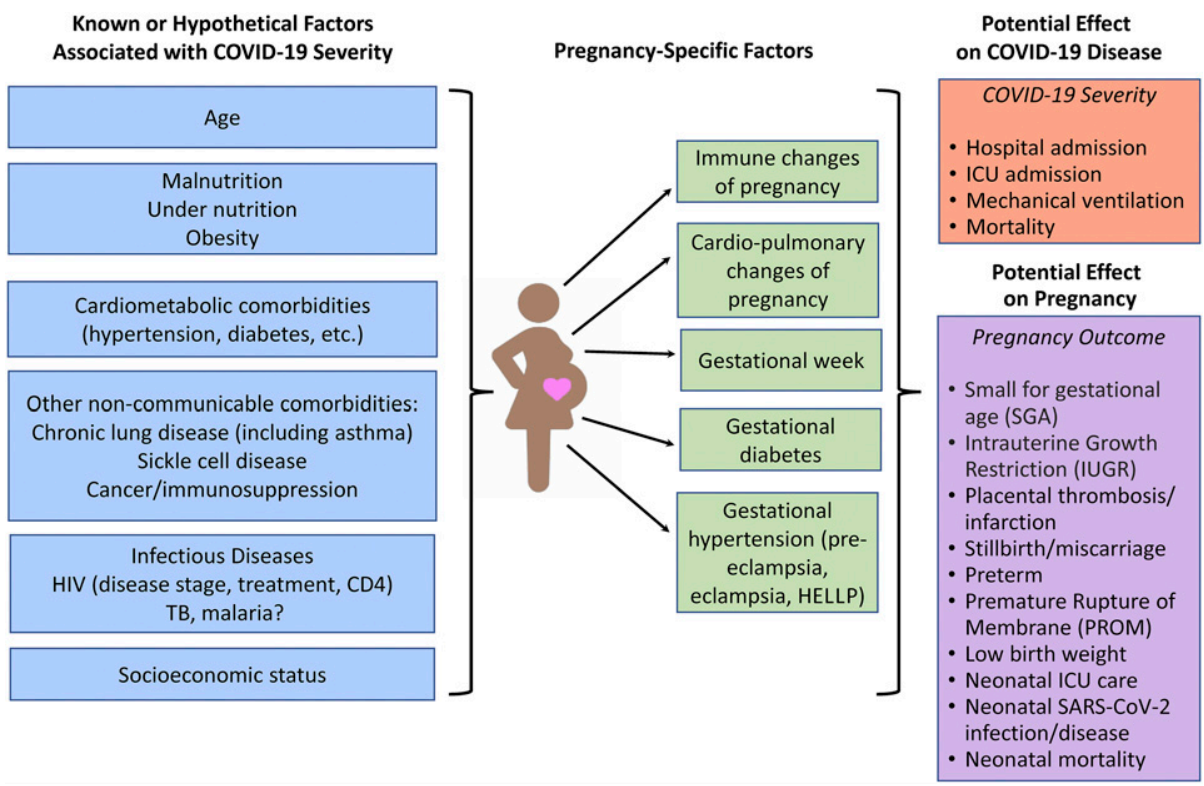

FIGURE 1. Known or hypothetical factors associated with COVID-19 disease, including pregnancy-specific factors, and effect on COVID-19 disease severity and potential effects on pregnancy outcome. HELLP = hemolysis, elevated liver enzymes, low platelets syndrome. 
outcomes for infants born to mothers with COVID-19 appear good, and reports of COVID-19-attributable neonatal mortality are rare. ${ }^{13,22}$ These studies are primarily from China and high-resource countries such as the United States and United Kingdom. No study has yet addressed the effect of maternal COVID-19 in the first trimester on the developing fetus. There are a few case reports of miscarriage with SARS-CoV-2 infection in early pregnancy, but there are also reports of women with infection early in gestation who recover and have healthy, term infants. ${ }^{23-25}$

Data on the effects of maternal SARS-CoV-2 infection on the fetus/neonate are limited, partly because of the lack of collection of appropriate laboratory specimens. In utero mother-to-child transmission of SARS-CoV-2 appears rare. ${ }^{26}$ In a meta-analysis of 176 published cases of laboratoryconfirmed SARS-CoV-2 infection in neonates, $12.2 \%$ of infections were thought to have occurred in utero (with only $5.7 \%$ having sufficient information to be classified as confirmed), $17.3 \%$ peripartum (with only $3.3 \%$ confirmed), and the majority of cases $(70.5 \%)$ were classified as postnatal infection because of environmental exposure. ${ }^{26}$ Determination of in utero mother-to-child SARS-CoV-2 transmission requires appropriate samples obtained with proper timing, including amniotic fluid, placenta, neonatal blood, and nasopharyngeal and other samples from the infant at birth. Persistence of infection in the neonate should also be documented. However, collection of the needed tissues and fluids and/or data on timing of infant testing has not been provided in many studies to date and may not be routinely collected during clinical care, particularly in low- and middle-income countries because of limited laboratory testing capacity and other logistical challenges. In the Raschetti et al. ${ }^{26}$ metaanalysis, neonatal symptoms were observed in only $55 \%$ of SARS-CoV-2 PCR-positive neonates, and it may be difficult to distinguish symptoms because of prematurity from those due to SARS-CoV-2; there were only three neonatal deaths (2.5\%), none of which were secondary to SARS-CoV-2 infection. Although SARS-CoV-2 has been on rare occasion identified by RT-PCR in breast milk, SARS-CoV-2 antibodies also have been documented, but the infectivity of the virus in breast milk has not been confirmed. ${ }^{27-29}$ Transmission of SARS-CoV-2 via breast milk has not been demonstrated to date. ${ }^{28}$

Although there are a number of registries that will target evaluation of the effects of SARS-CoV-2 infection in pregnant women and their neonates, they are predominantly in highincome settings (e.g., the UK Obstetric Surveillance System; the Pregnancy Coronavirus Outcomes Registry (PRIORITY) in the United States; the "MotherToBaby Pregnancy Studies" in the United States and Canada; the International Registry of Coronavirus Exposure in Pregnancy led by the United States and United Kingdom); none are focused on SSA. ${ }^{23,30-32}$ Given the paucity of data on SARS-CoV-2 and pregnancy from SSA, there is an urgent need to conduct surveillance and prospective studies across the region. Furthermore, retrospective analyses of routinely collected data are important, to assess the burden of SARS-CoV-2 infection among pregnant women (likely reflecting disease burden in the community) and to understand whether and how COVID-19 manifests differently among African women with HIV, TB, malaria, and other coinfections/comorbidities endemic to SSA. Comparisons of risk factors and outcomes can also be made across SSA countries and subregions while strengthening much-needed
intra-African south-to-south multi-disciplinary and interprofessional research collaborations. The African Forum for Research and Education in Health's ${ }^{33}$ COVID-19 Research Working Group has embarked upon such a collaboration across Westem, Central, Eastern, and Southem Africa.

Finally, despite the fact that pregnant women appear to be at higher risk of morbidity and mortality from COVID-19 than age-matched, nonpregnant women, pregnant women have been excluded from SARS-CoV-2 vaccine trials to date. ${ }^{34}$ Two SARS-CoV-2 mRNA-based vaccines have been reported to have high efficacy in reducing infections, with both already having received emergency use authorization by the UK Medicines and Healthcare Regulatory Agency and the U.S. Food and Drug Administration. ${ }^{35,36}$ Given the preponderance of women of reproductive age who work in health care (particularly in SSA), an area of profession that will be prioritized for initial phases of vaccine implementation, it is likely that some of these women will be pregnant, and others may be pregnant but not know it at the time of vaccination. Systems will need to be developed to track maternal/infant outcomes of COVID-19 vaccination in such pregnant women, which will be particularly challenging in SSA settings with weak health (especially in pregnancy) monitoring systems. As COVID-19 vaccines are rolled out globally, it is important to develop collaborative partnerships to enable surveillance of maternal/infant outcomes in SSA.

In the context of a global pandemic, vulnerable populations such as pregnant women and their infants are often neglected. This was observed in the initial years of the HIV epidemic and again with the Ebola virus, when clinical trials for drug and vaccine interventions for these high-fatality diseases did not include pregnant women, despite the high mortality in women and their infants. ${ }^{37}$ It is critical during the SARS-CoV-2 pandemic that we learn from prior experiences and include pregnant women and their infants in studies to 1) better understand the extent of infection and disease in this population and the effects on maternal/child health and 2) ensure consideration of pregnant women, as new treatments and vaccines to combat SARS-CoV-2 are being developed.

Received December 6, 2020. Accepted for publication December 17, 2020.

Published online December 28, 2020.

Acknowledgments: We would like to thank all the members of the AFREhealth Research Collaborative Working Group on COVID-19 as well as the continued support of the AFREhealth Executive Secretariat in Kumasi, Ghana (Ireneous N. Dasoberi, Clara Sam-Woode, and Georgina Yeboah). AFREhealth is a pan-African organization that seeks to work with Ministries of Health, training institutions and other stakeholders to improve the quality of healthcare in Africa through research, education, and capacity-building (www.afrehealth.org). The American Society of Tropical Medicine and Hygiene has waived the Open Access fee for this article due to the ongoing COVID-19 pandemic.

Disclosures: J.B.N. is an infectious disease internist and epidemiologist and Principal Investigator of the $\mathrm{NIH} / \mathrm{FIC}$ grant numbers 1R25TW011217-01 (African Association for Health Professions Education and Research); 1R21TW011706-01 (Cardiometabolic Outcomes, Mechanisms, and approach to prevention of Dolutegravir Associated Weight Gain in South Africa); and 1D43TW010937-01A1 (the University of Pittsburgh HIV-Comorbidities Research Training Program in South Africa). He is Chairman of AFREhealth Research committee and serves on the scientific program committee of the American Society of Tropical Medicine and Hygiene (ASTMH), as well as a senior fellow alumnus of the European Developing Countries Clinical Trial Partnership (EDCTP). N. A. S.-A. is a clinician-scientist 
and implementation researcher in Pediatric Infectious Diseases, and is supported by the NIH National Institute of Child Health and Human Development (NICHD) grant R01HD089866, and by an NIH/FIC award under the Adolescent HIV Prevention and Treatment Implementation Science Alliance (AHISA), for the Central and West Africa Implementation Science Alliance (CAWISA). Professor J.M.K., Associate Professor D.J.M.L., D.K.I.; M.T.P. and C.B.P.N are members of the DRC Ministry of Health's COVID-19 Multi-Sectoral Response Committee led by Professor J.J.M.T., who helped discover the Ebola virus in 1976. Sir Zumla is a co-PI of the Pan-African Network on Emerging and Re-Emerging Infections (PANDORA-ID-NET-https://www.pandoraid.net/) funded by the EDCTP, the EU Horizon 2020 Framework Program for Research and Innovation. Professor D.M.M. is a Congolese gynecologist and founder of the Panzi Hospital in eastern DRC where has treated thousands of women who were victims of rape as a weapon of war since. He was awarded the 2018 Nobel Peace Prize for his efforts to end the use of sexual violence as a weapon of war and armed conflict. Professor L.W.U. is the Chairman of the Epidemic Response Committee, Ahmadu Bello University Teaching Hospital, Zaria, Nigeria. All other co-authors have no conflicts of interest to declare. The content is solely the responsibility of the authors and does not necessarily represent the official views of the National Institutes of Health.

Authors' addresses: Jean B. Nachega, Health Sciences, Stellenbosch University, Cape Town, South Africa, Department of Epidemiology, Infectious Diseases and Microbiology, University of Pittsburgh Graduate School of Public Health, Pittsburgh, PA, and Department of Epidemiology, Johns Hopkins Bloomberg School of Public Health, Baltimore, MD, and Department of Medicine, Faculty of Medicine, E-mail: jnachega@sun.ac.za. Nadia A.Sam-Agudu, Institute of Human Virology Nigeria, Department of Pediatrics and International Research Center of Excellence, Abuja, Nigeria, Department of Pediatrics, Institute of Human Virology, University of Maryland School of Medicine, Baltimore, MD, and Department of Pediatrics and Child Health, School of Medical Sciences, University of Cape Coast, Cape Coast, Ghana, E-mail: nsamagudu@ihvnigeria.org. Samantha Budhram, Department of Obstetrics and Gynecology, University of KwaZulu Natal, Durban, South Africa, E-mail: budhrams@icloud.com. Taha E. Taha, Department of Epidemiology, Johns Hopkins Bloomberg School of Public Health, Baltimore, MD, E-mail: ttaha1@jhu.edu. Valerie Vannevel, UP/SAMRC Maternal and Infant Health Care Strategies Unit, Department of Obstetrics and Gynecology, Kalafong Hospital, University of Pretoria, Pretoria, South Africa, E-mail: valerie.vannevel@ up.ac.za. Priya Somapillay, Maternal Foetal Medicine, Steve Biko Hospital, Faculty of Health Sciences, University of Pretoria, Pretoria, South Africa, E-mail: priya.somapillay@up.ac.za. Daniel Katuashi Ishoso, Department of Community Health, Faculty of Medicine, Kinshasa School of Public Health, University of Kinshasa, Kinshasa, Democratic Republic of the Congo, E-mail: dishosok@gmail.com. Michel Tshiasuma Pipo, Department of Public Health, Centre Interdisciplinaire de Recherche en Ethnopharmacologie, Université Notre Dame du Kasayi, Kinshasa, Democratic Republic of Congo, E-mail: mycky1974@gmail.com. Christian Bongo-Pasi Nswe, Department of Public Health, Centre Interdisciplinaire de Recherche en Ethnopharmacologie, Université Notre-Dame du Kasayi Faculté de Médecine, Kananga, Democratic Republic of Congo, and Faculty of Public Health, Université Moderne de Kinkole, Kananga, Democratic Republic of Congo, E-mail: bongopanoudji@gmail.com. John Ditekemena, Department of Community Health, Faculty of Public Health, University of Kinshasa, Kananga, Democratic Republic of Congo, and Maternal and Child Health, Elisabeth Glaser Pediatric AIDS Foundation in Cameroon, Douala, Cameroon, E-mail: john.ditekemena@ unikin.ac.cd. Birhanu T. Ayele, Division of Epidemiology and Biostatistics, Department of Global Health, Faculty of Medicine and Health Sciences, Stellenbosch University, Cape Town, South Africa, E-mail: bayele@sun.ac.za. Rhoderick Machekano, Epidemiology and Biostatistics Unit, Faculty of Medicine and Health Sciences, African Center of Biostatistics Excellence (ACBE), Stellenbosch University, Cape Town, South Africa, E-mail: rhoderick@sun.ac.za. Onesmus W. Gachuno, Department of Obstetrics and Gynaecology, University of Nairobi, Nairobi, Kenya, E-mail: owgachuno@yahoo.com. JeanJacques Muyembe Tamfum, Department of Virology, National Instute of Bio-Medical Research (INRB), Kananga, Democratic Republic of Congo, and Department of Medical Microbiology and Virology, Faculty of Medicine, University of Kinshasa, Kananga,
Democratic Republic of Congo, E-mail: jjmuyembet@gmail.com. John Kinuthia, Department of Obstetrics and Gynaecology, University of Nairobi, Nairobi, Kenya, and Department of Research, Department of Reproductive Health, Kenyatta National Hospital, Nairobi, Kenya, E-mail: kinuthia@uw.edu. Nancy Mwongeli, Department of Research, Department of Reproductive Health, Kenyatta National Hospital, Nairobi, Kenya, E-mail: nancym390@ gmail.com. Musa Sekikubo, Department of Obstetrics and Gynaecology, School of Medicine, College of Health Sciences, Makerere University, Kampala, Uganda, E-mail: msekikubo@yahoo.com. Philippa Musoke, Department of Paediatrics and Child Health, School of Medicine, College of Health Sciences, Makerere University, Kampala, Uganda, E-mail: pmusoke@mujhu.org. Evans Kofi Agbeno, Department of Obstetrics and Gynecology, Cape Coast Teaching Hospital, University of Cape Coast, Cape Coast, Ghana, E-mail: evans.agbeno@ucc.edu.gh. Lawal W. Umar, Department of Pediatrics, College of Health Sciences, Ahmadu Bello Teaching Hospital, Ahmadu Bello University, Zaria, Nigeria, E-mail: umarlw@ gmail.com. Mukanire Ntakwinja and Denis M. Mukwege, Department of Obstetrics and Gynecology, Panzi Hospital, Bukavu, Democratic Republic of the Congo, and Department of Obstetrics and Gynecology, Universite Evangelique en Afrique, Bukavu, Democratic Republic of the Congo, E-mails: bmukanire@yahoo.fr and denismukwege@hotmail.com. Emily R. Smith, Department of Global Health, Milken Institute School of Public Health, George Washington University, Washington, DC, E-mail: emilysmith@email.gwu.edu. Eduard J. Mills, Department of Health Research Evidence and Impact, Faculty of Health Sciences, McMaster University, Hamilton, Canada, E-mail: emills@mteksciences.com. John Otokoye Otshudiema, Epidemiological Surveillance Team, COVID-19 Response, Health Emergencies Program, World Health Organization, Kinshasa, Democratic Republic of the Congo, E-mail: johnotokoye@gmail.com. Placide Mbala-Kingebeni, Microbiology and Virology, Institut National de Recherche Biomedicale, Kinshasa, Democratic Republic of the Congo, and Department of Medical Microbiology and Virology, Faculty of Medicine, E-mail: mbalaplacide@gmail.com. Jean-Marie N. Kayembe, Department of Medicine, Faculty of Medicine, University of Kinshasa, Kinshasa, Democratic Republic of the Congo, E-mail: jm.kayembe@ unikin.ac.cd. Don Jethro Mavungu Landu, Department of Public Health, Centre Interdisciplinaire de Recherche en Ethnopharmacologie, Faculty of Medicine, Université Notre Dame du Kasayi, Kinshasa, Democratic Republic of the Congo, E-mail: jethromavungu@gmail.com. Alimuddin Zumla, Division of Infection and Immunity, Department of Infection, University College London, London, United Kingdom, and National Institute for Health Research Biomedical Research Centre, University College London Hospitals NHS Foundation Trust, University College London Hospitals NHS Foundation Trust, London, United Kingdom, E-mail: a.zumla@ucl.ac.uk. Eduard J. Langenegger, Department of Obstetrics and Gynecology, Faculty of Medicine and Health Sciences, Stellenbosch University, Tyberberg Teaching Hospital, Cape Town, South Africa, E-mail: langen@sun.ac.za. Lynne M. Mofenson, Elizabeth Glaser Pediatric AIDS Foundation, Washington DC, E-mail: mofensol@ gmail.com.

This is an open-access article distributed under the terms of the Creative Commons Attribution (CC-BY) License, which permits unrestricted use, distribution, and reproduction in any medium, provided the original author and source are credited.

\section{REFERENCES}

1. World Health Organization, 2020. Maternal and NewbornMortality/Causes of Death. Available at: https://www.who.int/ data/maternal-newborn-child-adolescent-ageing/maternaland-newborn-data/maternal-and-newborn---mortality-causesof-death. Accessed December 4, 2020.

2. Coker M, Folayan MO, Michelow IC, Oladokun RE, Torbunde N, Sam-Agudu NA, 2020. Things must not fall apart: the ripple effects of the COVID-19 pandemic on children in sub-Saharan Africa. Pediatr Res. doi: 10.1038/s41390-020-01174-y.

3. World Health Organization, 2020. Coronavirus Disease (COVID-19) Dashboard. Available at: https://covid19.who. int/?gclid=EAlalQobChMImpz1vPGt7QIVdIBQBh3XIAiTEA AYASAAEgJR8_D_BwE. Accessed December 1, 2020. 
4. Fairlie L et al., 2020. COVID-19 in pregnancy in South Africa: tracking the epidemic and defining the natural history. S Afr Med J 110: 729-731.

5. Siston AM et al., 2010. Pandemic 2009 influenza $A(H 1 N 1)$ virus illness among pregnant women in the United States. JAMA 303: 1517-1525.

6. Mertz D, Geraci J, Winkup J, Gessner BD, Ortiz JR, Loeb M, 2017. Pregnancy as a risk factor for severe outcomes from influenza virus infection: a systematic review and meta-analysis of observational studies. Vaccine 35: 521-528.

7. Ellington S, Strid P, Tong VT, Woodworth K, Galang RR, Zambrano LD, Nahabedian J, Anderson K, Gilboa SM, 2020. Characteristics of women of reproductive age with laboratoryconfirmed SARS-CoV-2 infection by pregnancy status - United States, January 22-June 7, 2020. MMWR Morb Mortal Wkly Rep 69: 769-775.

8. Yu N et al., 2020. Clinical features and obstetric and neonatal outcomes of pregnant patients with COVID-19 in Wuhan, China: a retrospective, single-centre, descriptive study. Lancet 20: 559-564.

9. Sutton D, Bertozzi-Villa C, Lasky J, Fuchs K, Friedman A, 2020. Outcomes and epidemiology of COVID-19 infection in the obstetric population. Semin Perinatol 44: 151283.

10. Khalil A et al., 2020. SARS CoV 2 infection in pregnancy: a systematic review and meta-analysis of clinical features and pregnancy outcomes. EClinical Med 25: 100446.

11. Blitz MJ, Rochelson B, Rausch AE, Solmonovich R, Shan W, Combs A, Nimaroff M, 2020. Universal testing for coronavirus disease 2019 in pregnant women admitted for delivery: prevalence of peripartum infection and rate of asymptomatic carriers at four New York hospitals within an integrated healthcare system. Am J Obstet Gynecol MFM 2: 100169.

12. Abeysuriya $S$, Wasif $S$, Counihan $C$, Shah N, lliodromiti S, CutinoMoguel MT, Saeed F, Velauthar L, 2020. Universal screening for SARS-CoV-2 in pregnant women at term admitted to an East London maternity unit. Eur J Obstet Gynecol Repro Biol 252: 444-446.

13. Allotey $\mathrm{J}$ et al., 2020. Coronavirus disease (COVID-19) in pregnancy: a living systematic review on clinical manifestations, risk factors and maternal and perinatal outcomes. BMJ 370: $\mathrm{m} 3320$.

14. Zambrano LD et al., 2020. Update: characteristics of symptomatic women of reproductive age with laboratory-confirmed SARSCoV-2 infection by pregnancy status, United States, January 22-October 3, 2020. MMWR Morb Mortal Wkly Rep 69: 1641-1647.

15. Breslin $\mathrm{N}$ et al., 2020. Coronavirus disease 2019 infection among asymptomatic and symptomatic pregnant women: two weeks of confirmed presentations to an affiliated pair of New York city hospitals. Am J Obstet Gynecol MFM 2: 100118.

16. Wadman $M, 2020$. Why infection poses a special risk to pregnant women. Science 369: 606-607.

17. Blitz MJ et al., 2020. Maternal mortality among women with coronavirus disease 2019 admitted to the intensive care unit. Am J Obstet Gynecol 223: 595-599.

18. Ngalame AN et al., 2020. Materno-fetal outcomes of COVID-19 infected pregnant women managed at the Douala GynecoObstetric and Pediatric Hospital-Cameroon. Open J Obstet Gynecol 10: 1279-1294.

19. Aziz Diouf A et al., 2020. Clinical characteristics and outcomes of COVID-19 infection in nine pregnant women: a report from a sub-Saharan African country, Senegal. Pan Africa Med J 35: 58.

20. Nachega JB et al., 2020. Clinical characteristics and outcomes of patients hospitalized for COVID-19 in Africa: early insights from the Democratic Republic of the Congo. Am J Trop Med Hyg 103: 2419-2428.

21. Khoury R et al., 2020. Characteristics and outcomes of 241 births to women with severe acute respiratory syndrome coronavirus 2 (SARS-CoV-2) infection at five New York city medical centers. Obstet Gynecol 136: 273-282.

22. Woodworth KR et al., 2020. Birth and infant outcomes following laboratory-confirmed SARS-CoV-2 infection in pregnancy SET-NET, 16 jruisdictions, March 29-October 14 2020. MMWR Morb Mortal Wkly Rep 69: 1635-1640.

23. Baud D, Greub G, Favre G, Gengler C, Jaton K, Dubruc E, Pomar $\mathrm{L}, 2020$. Second-trimester miscarriage in a pregnant woman with SARS-CoV-2 infection. JAMA 323: 2198-2200.

24. Da Silva JV, Silva KSC, dos Santos VE, 2020. SARS-CoV-2 in the first trimester of pregnancy: potential interference in placentation. J Matern Fetal Neonatal Med. doi: 10.1080/14767058. 2020.1792879

25. Knight $M$ et al., 2020. Characteristics and outcomes of pregnant women admitted to the hospital with confirmed SARS-CoV-2 infection in UK: national population-based cohort study. BMJ 369: $\mathrm{m} 2107$.

26. Raschetti R, Vivanti AJ, Vauloup-Fellous C, Loi B, Benachi A, De Luca D, 2020. Synthesis and systematic review of reported neonatal SARS-CoV-2 infections. Nat Commun 11: 5164

27. Chambers $\mathrm{C}$, Krogstad $\mathrm{P}$, Bertrand K, Contreras D, Tobin NH, Bode L, Aldrovandi G, 2020. Evaluation for SARS-CoV-2 in breast milk from 18 infected women. JAMA 324: e2015580.

28. Centreno-Tablante E, Medina-Rivera M, Finkelstein JL, RaycoSolon P, Garcia-Casal MN, Rogers L, Ghezzi-Kopel K, Ridwan $P$, Peña-Rosas JP, Mehta S, 2020. Transmission of SARS-CoV2 through breast milk and breastfeeding: a living systematic review. Ann N Y Acad Sci. doi: 10.1111/nyas.14477.

29. Fox A, Marino J, Amanat F, Krammer F, Hahn-Holbrook F, ZollaPazner S, Powell RL, 2020. Robust and specific secretory IgA against SARS-CoV-2 detected in human milk. iScience 23: 101735.

30. PRIORITY, 2020. PRIORITY: Pregnancy Coronavirus Outcomes Registry. Available at: https://priority.ucsf.edu/about-priority. Accessed December 4, 2020.

31. Organization Technology Information System National Office, 2020. MothertoBaby COVID-19 and Pregnancy Study. Available at: https://mothertobaby.org/ongoing-study/coronaviruscovid-19/. Accessed December 4, 2020.

32. IRCP, 2020. Intemational Registry of Coronavinus Exposure in Pregnancy. Available at: https://corona.pregistry.com/. Accessed December 4, 2020.

33. Omaswa $\mathrm{F}$ et al., 2017. Medical education partnership initiative gives birth to AFREhealth. Lancet Glob Health 5: e965-e966.

34. Heath PT, Le Doare K, Khalil A, 2020. Inclusion of pregnant women in COVID-19 vaccine development. Lancet Infect Dis 20: 1007-1008.

35. The New York Times, 2020. UK Approves Pfizer Coronavirus Vaccine, a First in the West. Available at: https://www.nytimes.com/ 2020/12/02/world/europe/pfizer-coronavirus-vaccine-approveduk.html. Accessed December 4, 2020.

36. FDA, 2020. FDA Takes Key Action in Fight against COVID-19 by Issuing Emergency Use Authorization for First COVID-19 Vaccine. Available at: https://www.fda.gov/news-events/pressannouncements/fda-takes-key-action-fight-against-covid-19issuing-emergency-use-authorization-first-covid-19. Accessed December 16, 2020.

37. Gomes MF, de la Fuente-Nunez V, Saxena A, Kuesel AC, 2017. Protected to deaths: systematic exclusion of pregnant women from Ebola virus disease trials. Reprod Health 14 (Supp/3): 172. 